\title{
Bilateral pleural effusions associated with pravastatin sodium treatment
}

\section{To the Editors:}

Pravastatin sodium is a 3-hydroxyl-3-methylglutaryl-CoA reductase blocker used in the treatment of hypercholesterolaemia. We report on a patient who developed bilateral pleural effusions while he was being treated with the drug. The patient was a 60-yr-old male, a nonsmoker who was under treatment with $40 \mathrm{mg} \cdot$ day $^{-1}$ pravastatin since September 2005. He had no history of allergy or significant environmental or occupational exposure to agents that may harm the respiratory system.

He presented with persistent bilateral pleuritic chest pain and mild dyspnoea of several days' duration in September 2006. He had neither constitutional symptoms nor extrathoracic abnormalities. A chest radiograph revealed small bilateral pleural effusions with predominance on the left side. Computed tomography (CT) with pulmonary angiography algorithm showed no intravascular clot, confirmed the presence of pleural fluid (PF), especially on the left side, and further revealed bilateral pleural thickening.

Blood tests were only significant for an elevated C-reactive protein $\left(13.6 \mathrm{mg} \cdot \mathrm{L}^{-1}\right.$; upper limit $\left.<6 \mathrm{mg} \cdot \mathrm{L}^{-1}\right)$. Serum antinuclear antibodies were negative. A diagnostic thoracentesis on the left side revealed a serosanguinious fluid of $\mathrm{pH} 7.38$. Other features of the fluid were as follows: lactate dehydrogenase $263 \mathrm{IU} \cdot \mathrm{L}^{-1}(\mathrm{PF} /$ serum ratio: 0.8$)$; protein $4.2 \mathrm{mg} \cdot \mathrm{dL}^{-1}$ (PF/serum ratio: 0.55 ); glucose $98 \mathrm{mg} \cdot \mathrm{dL}^{-1}$; and nucleated cell count 1,100 cells $\mu \mathrm{L}^{-1}$ (of which lymphocytes $84 \%$, neutrophils $14 \%$ and eosinophils $2 \%$ ). The PF cytology and microbiology studies were negative. PF adenosine deaminase was $25 \mathrm{U} \cdot \mathrm{L}^{-1}$. Ultrasound studies of the heart and lower limbs were normal. Bronchoscopy disclosed no endobronchial abnormality. A CTguided fine needle biopsy of the thickened pleura showed fibrotic and nonspecific inflammatory changes. Since the pleural effusions persisted, pravastatin was withdrawn in October 2006. After discontinuation of the drug, the symptoms resolved and C-reactive protein levels fell to normal and, in a thoracic CT obtained in January 2007, the patient had residual pleural thickening with no evidence of PF in both hemithoraces. He was last seen in June 2007, when he was asymptomatic with no re-accumulation of the PF. Therefore, we suggest that the failure to establish a specific cause of the pleural effusions despite an extensive diagnostic work-up and the rapid improvement after the discontinuation of the drug strongly suggest the presence of an aetiologic link between pravastatin and the pleural effusions.

Pleural effusions which appeared 6 months to 13 yrs after the initiation of therapy had been previously attributed to treatment with simvastatin, another member of the statin family [1, 2], but to the best of the present authors' knowledge, this is the first case of pleural effusions caused by pravastatin. The patient who developed pleural effusions during treatment with simvastatin had peripheral and pleural fluid or bronchoalveolar lavage eosinophilia, interstitial lung fibrosis and abnormal liver function or weight loss; however involvement of extrapleural sites and generalised symptoms were absent in the patient reported here. This suggests that different mechanisms are most likely responsible for pleural disease occurring as an adverse effect of the statins.

To conclude, pravastatin along with simvastatin, and possibly other statins, must be considered as a cause of pleural effusion of indeterminate aetiology. Since relevant clinical syndromes are widely heterogeneous, resolution of the disease after drug discontinuation is the only way to establish the diagnosis of a statin-associated pleural effusion.

\section{Kalomenidis, S. Papiris and S. Loukides}

2nd Dept of Pulmonary Medicine, Athens Medical School, "Attikon" Hospital, Haidari, Greece.

\section{STATEMENT OF INTEREST}

None declared.

\section{REFERENCES}

1 De Groot RE, Willems LN, Dijkman JH. Interstitial lung disease with pleural effusion caused by simvastin. I Intern Med 1996; 239: 361-363.

2 Roncato-Sabéran M, Hustache-Mathieu L, Hoen B. Eosinophilic pleural effusion caused by simvastatin after 13 years of exposure. Eur J Intern Med 2006; 17: 450.

DOI: $10.1183 / 09031936.00087007$ 